\title{
Detection of a flow induced magnetic field eigenmode in the Riga dynamo facility
}

\author{
Agris Gailitis, Olgerts Lielausis, Sergej Dement'ev, Ernests Platacis, Arnis Cifersons \\ Institute of Physics, Latvian University \\ LV-2169 Salaspils 1, Riga, Latvia \\ Gunter Gerbeth, Thomas Gundrum, Frank Stefani \\ Forschungszentrum Rossendorf \\ P.O. Box 510119, D-01314 Dresden, Germany \\ Michael Christen, Heiko Hänel, Gotthard Will \\ Dresden University of Technology, Dept. Mech. Eng. \\ P.O. Box 01062, Dresden, Germany \\ (Submitted to Phys. Rev. Lett., December 10, 1999)
}

In an experiment at the Riga sodium dynamo facility, a slowly growing magnetic field eigenmode has been detected over a period of about 15 seconds. For a slightly decreased propeller rotation rate, additional measurements showed a slow decay of this mode. The measured results correspond satisfactory with numerical predictions for the growth rates and frequencies.

PACS numbers: 47.65.+a, 52.65.Kj, 91.25.Cw

Magnetic fields of cosmic bodies, such as the Earth, most of the planets, stars and even galaxies are believed to be generated by the dynamo effect in moving electrically conducting fluids. Whereas technical dynamos consist of a number of well-separated electrically conducting parts, a cosmic dynamo operates, without any ferromagnetism, in a nearly homogeneous medium (for an overview see, e.g., [1] and [2]).

The governing equation for the magnetic field $\mathbf{B}$ in an electrically conducting fluid with conductivity $\sigma$ and the velocity $\mathbf{v}$ is the so-called induction equation

$$
\frac{\partial \mathbf{B}}{\partial t}=\operatorname{curl}(\mathbf{v} \times \mathbf{B})+\frac{1}{\mu_{0} \sigma} \Delta \mathbf{B}
$$

which follows from Maxwell equations and Ohms law. The obvious solution $\mathbf{B}=\mathbf{0}$ of this equation may become unstable for some critical value $R m_{c}$ of the magnetic Reynolds number

$$
R m=\mu_{0} \sigma L v
$$

if the velocity field fulfills some additional conditions. Here $L$ is a typical length scale, and $v$ a typical velocity scale of the fluid system. $R m_{c}$ depends strongly on the flow topology and the helicity of the velocity field. For self-excitation of a magnetic field it has to be at least greater than one. For typical dynamos as the Earth outer core, $R m$ is supposed to be of the order of 100 .

The last decades have seen an enormous progress of dynamo theory which deals, in its kinematic version, with the induction equation exclusively or, in its full version, with the coupled system of induction equation and Navier-Stokes equation for the fluid motion. Numerically, this coupled system of equations has been treated for a number of more or less realistic models of cosmic bodies (for an impressive simulation, see [3]).

Quite contrary to the success of dynamo theory, experimental dynamo science is still in its infancy. This is mainly due to the large dimensions of the length scale and/or the velocity scale which are necessary for dynamo action to occur. Considering the conductivity of sodium as one of the best liquid conductors $\left(\sigma \approx 10^{7}(\Omega \mathrm{m})^{-1}\right.$ at $100^{\circ} \mathrm{C}$ ) one gets $\mu_{0} \sigma \approx 10 \mathrm{~s} / \mathrm{m}^{2}$. For a very efficient dynamo with a supposed $R m_{c}=10$ this would amount to a necessary product $L v=1 \mathrm{~m}^{2} / \mathrm{s}$ which is very large for a laboratory facility, even more if one takes into account the technical problems with handling sodium. Historically notable for experimental dynamo science is the experiment of Lowes and Wilkinson where two ferromagnetic metallic rods were rotated in a block at rest $[$. A first liquid metal dynamo experiment quite similar to the present one was undertaken by some of the authors in 1987. Although this experiment had to be stopped (for reasons of mechanical stability) before dynamo action occurred the extrapolation of the amplification factor of an applied magnetic field gave indication for the possibility of magnetic field self-excitation at higher pump rates [5]. Today, there are several groups working on liquid metal dynamo experiments. For a summary we refer to the workshop "Laboratory Experiments on Dynamo Action" held in Riga in summer 1998 [6].

After years of preparation and careful velocity profile optimization on water models, first experiments at the Riga sodium facility were carried out during November 6-11, 1999. The present paper comprises only the most important results of these experiments, one of them being the observation of a dynamo eigenmode slowly growing in time at the maximum rotation rate of the propeller. A more comprehensive analysis of all measured data will be published elsewhere. 


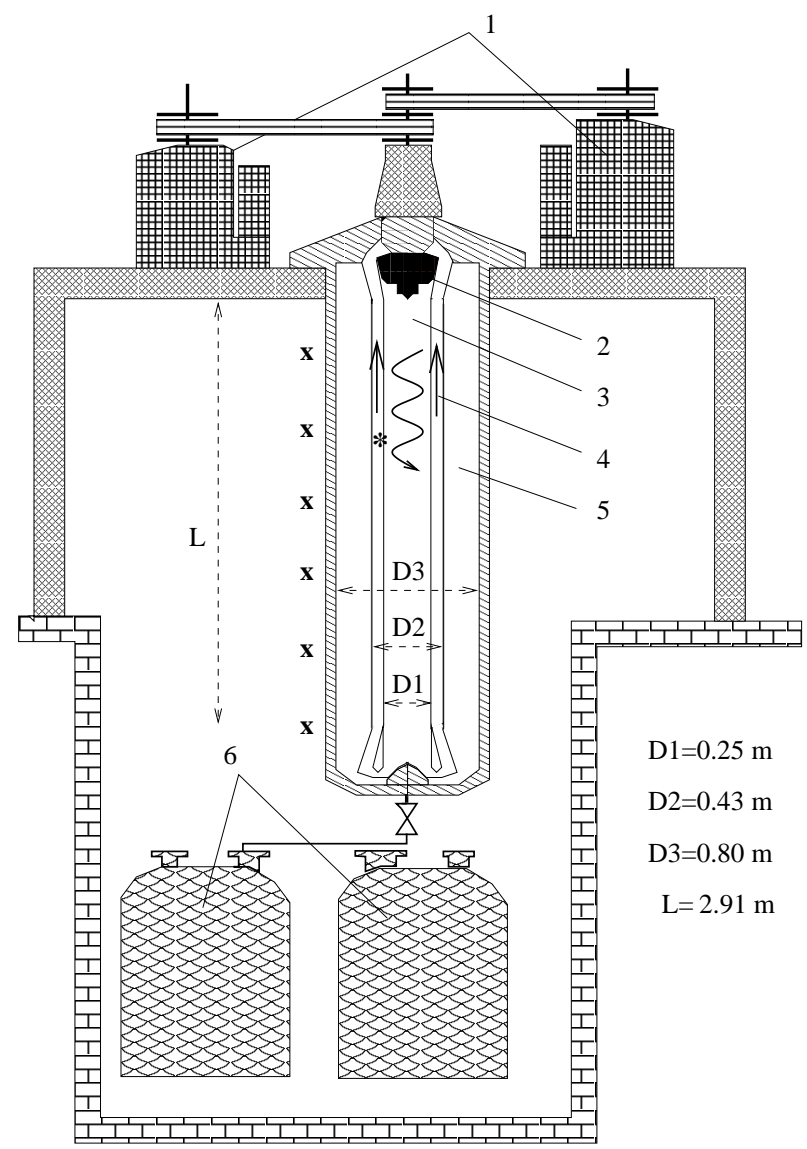

FIG. 1. The Riga dynamo facility. Main parts comprising: 1 - Two motors (55 kW each), 2 - Propeller, 3 - Helical flow region, 4 - Back-flow region, 5 - Sodium at rest, 6 - Sodium storage tanks, * - Position of the flux-gate sensor, $\times$ - Positions of the six Hall sensors.

The principal design of the dynamo facility, together with some of the most important dimensions, is shown in Fig. 1. The main part of the facility consists of a spiral flow of liquid sodium in an innermost tube (with a velocity up to the order of $15 \mathrm{~m} / \mathrm{s}$ ) with a coaxial backflow region and a region with sodium at rest surrounding it. The total amount of sodium is $2 \mathrm{~m}^{3}$. The sodium flow up to $0.6 \mathrm{~m}^{3} / \mathrm{s}$ is produced by a specially designed propeller which is driven by two $55 \mathrm{~kW}$ motors.

All three sodium volumes play an important role in the magnetic field generation process. The spiral flow within the immobile sodium region amplifies the magnetic field by stretching field lines [7]. The back-flow is responsible for a positive feedback [8]. The result is an axially non-symmetric field (in a symmetric flow geometry!) slowly rotating around the vertical axis. Hence, a low frequency $\mathrm{AC}$ magnetic field is expected for this configuration. Concerning the azimuthal dependence of the magnetic field which includes terms of the type $\exp (\operatorname{im} \varphi)$ with in general arbitrary $m$ it is well-known that for those $\mathrm{Rm}$ available in this experiment only the mode with $m=1$ can play any role [8]. A lot of details concerning the solution of the induction equation for the chosen experimental geometry and the optimization of the whole facility in general and of the shape of the velocity profiles in particular can be found in [8], [9], and [10].

For the magnetic field measurements we used two different types of sensors. Inside the dynamo, close to the innermost wall and at a height of $1 / 3$ of the total length from above, a flux-gate sensor was positioned. Additionally, 8 Hall sensors were positioned outside the facility at a distance of $10 \mathrm{~cm}$ from the thermal isolation. Of those, 6 were arranged parallel to the dynamo axis with a relative distance of $50 \mathrm{~cm}$, starting with $35 \mathrm{~cm}$ from the upper frame. Two sensors were additionally arranged at different angles.

After heating up the sodium to $300^{\circ} \mathrm{C}$ and pumping it slowly through the facility for 24 hours (to ensure good electrical contact of sodium with the stainless-steel walls) various experiments at $250^{\circ} \mathrm{C}$ and around $205^{\circ} \mathrm{C}$ at different rotation rates of the propeller were carried out.

According to our numerical predictions, self-excitation was hardly to be expected much above a temperature of $200^{\circ} \mathrm{C}$ since the electrical conductivity of sodium decreases significantly with increasing temperature. Nevertheless, we started experiments at $250^{\circ} \mathrm{C}$ in order to get useful information for the later dynamo behaviour at lower temperature, i.e. at higher $\mathrm{Rm}$. Although the experiment was intended to show self-excitation of a magnetic field without any noticeable starting magnetic field, kick-field coils fed by a 3-phase current of variable low frequency were wound around the module in order to measure the sub-critical amplification of the applied magnetic field by the dynamo. This measurement philosophy was quite similar to that of the 1987 experiment [11] and is based on generation theory for prolongated flows as the length of our spiral flow exceeds its diameter more then ten times. Generation in such a geometry should start as exponentially high amplification of some kick-field (known as convective generation) and should transform, at some higher flowrate, into self-excitation without any external kick-field.

As a typical example of a lot of measured field amplification curves, Fig. 2 shows the inverse relation of the measured magnetic field to the current in the excitation coils for a frequency of $1 \mathrm{~Hz}$ and a temperature of $205^{\circ} \mathrm{C}$ versus the rotation rate of the propeller. The two curves (squares and crosses) correspond to two different settings of the 3-phase current in the kick-field coils with respect to the propeller rotation. An increasing amplification of the kick-field can be clearly observed in both curves until a rotation rate of about $1500 \mathrm{rpm}$. These parts of both curves point to about $1700 \mathrm{rpm}$ which might be interpreted as the onset of convective generation [8], [9]. If the excitation frequency would be exactly the one the system likes to generate as its eigenmode, the curves should further approach the abscissa axis up to the self-excitation point. As $1 \mathrm{~Hz}$ does not meet exactly the eigenmode fre- 
quency the points are repelled from the axis for further increasing rotation rates as it is usual for externally excited linear systems passing the point of resonance (for this interpretation, see also Fig. 5).

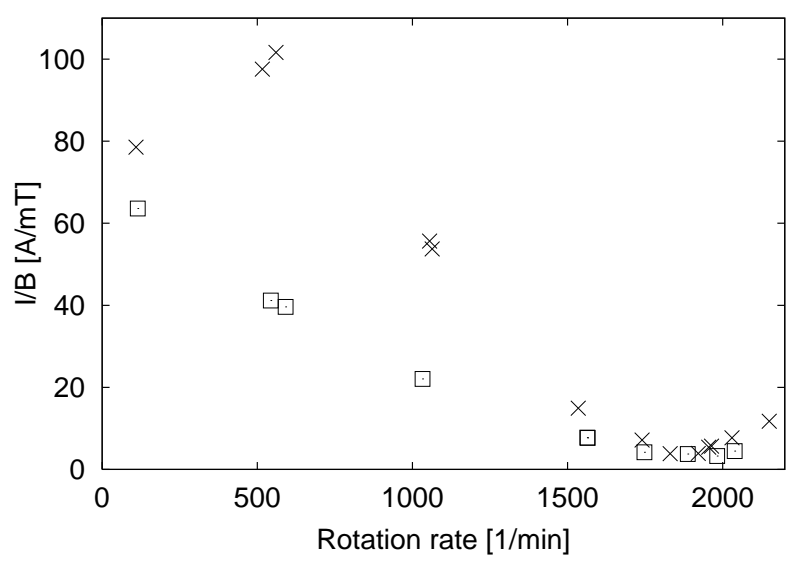

FIG. 2. Dependence of the magnetic field amplification on the propeller rotation rate for $\mathrm{T}=205^{\circ} \mathrm{C}$ and $\mathrm{f}=1 \mathrm{~Hz}$. The ordinate axis shows the inverse relation of the measured magnetic field to the current in the kick-field coils. Squares and crosses correspond to two different settings of the 3-phase current in the kick-field coils with respect to the propeller rotation.

It should be underlined that all points on Fig. 2 except the rightmost one are calculated from sinusoidal field records showing the same $1 \mathrm{~Hz}$ frequency as the kick-field. However, the rightmost point at $2150 \mathrm{rpm}$ is exceptional. Let us analyse the magnetic field signal at this rotation rate in more detail. Fig 3 a shows the magnetic field measured every $10 \mathrm{~ms}$ at the inner sensor in an interval of 15 s. Evidently, there is a superposition of two signals.

Numerically, this signal (comprising 1500 data points) has been analyzed by means of a non-linear least square fit with 8 free parameters according to

$$
B(t)=A_{1} e^{p_{1} t} \sin \left(2 \pi f_{1} t+\phi_{1}\right)+A_{2} e^{p_{2} t} \sin \left(2 \pi f_{2} t+\phi_{2}\right)
$$

The curve according to this ansatz (which is also shown in Fig. 3a) fits extremely well into the data giving the following parameters (the errors are with respect to a 68.3 per cent confidence interval):

$$
\begin{aligned}
A_{1} & =(0.476 \pm 0.004) \mathrm{mT}, \quad p_{1}=(-0.0012 \pm 0.0003) \mathrm{s}^{-1} \\
f_{1} & =(0.995 \pm 0.00005) \mathrm{s}^{-1}, \quad \phi_{1}=-0.879 \pm 0.012 \\
A_{2} & =(0.133 \pm 0.001) \mathrm{mT}, \quad p_{2}=(0.0315 \pm 0.0009) \mathrm{s}^{-1} \\
f_{2} & =(1.326 \pm 0.00015) \mathrm{s}^{-1}, \quad \phi_{2}=(0.479 \pm 0.009)
\end{aligned}
$$

The positive parameter $p_{2}=0.0315 s^{-1}$ together with the very small error gives clear evidence for the appearance of a self-exciting mode at the rotation rate of 2150 rpm. Fig. 3b shows in a decomposed form the two contributing modes, the larger one reflecting the amplified field of the coils and the smaller one reflecting the selfexcited mode.

(a)

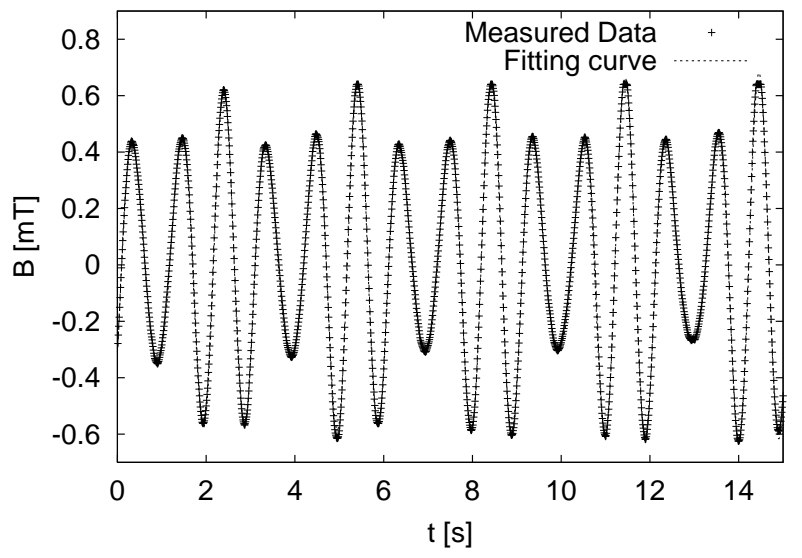

(b)

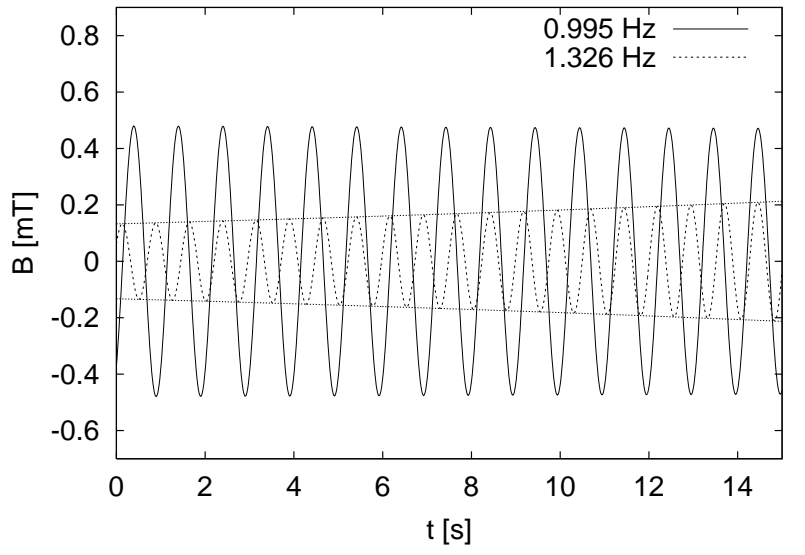

FIG. 3. Measured magnetic field and fitting curve (a). Decomposition of the fitting curve into two curves with different frequencies (b).

For reasons of some technical problems, this highest rotation rate could be hold only for 20 seconds after which it fell down to $1980 \mathrm{rpm}$. At that lower rotation rate the coil current was switched of suddenly. Figure 4 shows the magnetic field behaviour at three selected Hall sensors positioned outside the dynamo. This mode has a frequency of $f=1.1 \mathrm{~s}^{-1}$ and a decay rate of $p=-0.3 \mathrm{~s}^{-1}$. A similar signal was recorded by the inner fluxgate sensor, too.

It is interesting to compare the frequencies and growth or decay rates at the two different rotation rates $2150 \mathrm{rpm}$ and $1980 \mathrm{rpm}$ with the numerical predictions. These are based on the outcomes of a two-dimensional time dependent code which was described in [9]. As input velocity for the computations an extrapolated velocity field based on measurements in water at two different heights and at three different rotation rates (1000, 1600, and $2000 \mathrm{rpm}$ ) was used. Fig. 5 shows the predicted growth rates and frequencies for the three temperatures $150^{\circ} \mathrm{C}, 200^{\circ} \mathrm{C}$, and $250^{\circ} \mathrm{C}$ which are different due to the dependence of the electrical conductivity on temperature. The two pairs of 
points in Fig. 5 represent the respective measured values. Having in mind the limitations and approximations of the numerical prognosis [9] the agreement between precalculations and measured values is good, particularly regarding the frequencies of the magnetic field eigenmode.

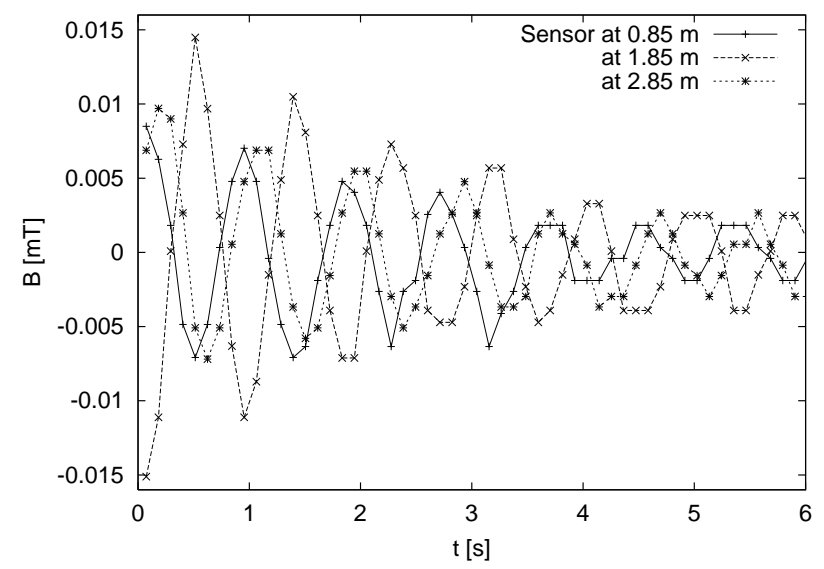

FIG. 4. Magnetic fields measured at 3 selected positions outside the dynamo module after switching off the coil current.

The main part of the experiment was originally planned at $\mathrm{T}=150^{\circ} \mathrm{C}$ where self-excitation with a much higher growth rate was expected. Unfortunately, the safety rules required to stop the experiment at $\mathrm{T}=205^{\circ} \mathrm{C}$ since technical problems with the seal of the propeller axis against the sodium flow-out have been detected. It is worth to be noted that the overall system worked stable and without problems over a period of about five days. The sealing problem needs inspection, but represents no principle problem.

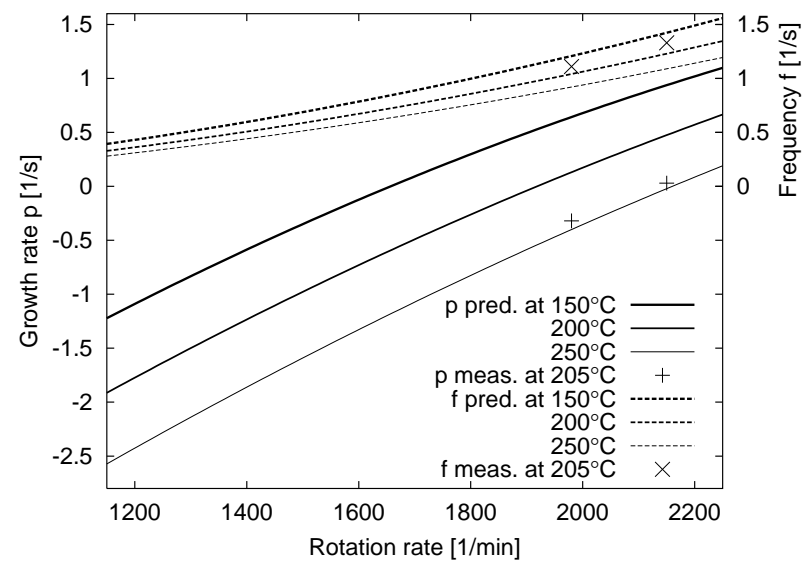

FIG. 5. Numerical predictions for growth rates $p$ and frequencies $f$ of the dynamo eigenmode in dependence on the rotation rate for three different temperatures, and measured values.

For the first time, magnetic field self-excitation was observed in a liquid metal dynamo experiment. Expectedly, the observed growth rate was still very small. The correspondence of the measured growth rates and frequencies with the numerical prognoses is convincing. The general concept of the experiment together with the fine-tuning of the velocity profiles [9] have been proven as feasible and correct. The facility has the potential to exceed the threshold of magnetic field self-excitation by some 20 per cent with respect to the critical magnetic Reynolds number. The experiment will be repeated at lower temperature when the technical problems with the seal will be resolved. For lower temperature, a higher growth rate will drive the magnetic field to higher values where the back-reaction of the Lorentz forces on the velocity should lead to saturation effects.

We thank the Latvian Science Council for support under grant 96.0276, the Latvian Government and International Science Foundation for support under joint grant LJD100, the International Science Foundation for support under grant LFD000 and Deutsche Forschungsgemeinschaft for support under INK 18/A1-1. We are grateful to $\mathrm{W}$. Häfele for his interest and support, and to the whole experimental team for preparing and running the experiment.

[1] F. Krause and K.-H. Rädler, Mean-field magnetohydrodynamics and dynamo theory, (Akademie-Verlag, Berlin, and Pergamon Press, Oxford, 1980)

[2] H. K. Moffat, Magnetic field generation in electrically conducting fluids, (Cambridge University Press, 1978)

[3] G. A. Glatzmeier and P. H. Roberts, Nature 377, 203 (1995)

[4] F. J. Lowes and I. Wilkinson, Nature 198, 1158 (1963)

[5] A. Gailitis, B. G. Karasev, I. R. Kirillov, O. A. Lielausis, S. M. Luzhanskii, A. P. Ogorodnikov, G. V. Preslitskii, Mag. Gidrodin., No.4, 3, (1987)

[6] Proceedings of the International Workshop on Laboratory Experiments on Dynamo Action, Riga, June 14-16, 1998, edited by O. Lielausis, A. Gailitis, G. Gerbeth, F. Stefani, (FZ Rossendorf, 1998)

[7] Yu. B. Ponomarenko, Zh. Prikl. Mekh. Tekhn. Fiz.(USSR) 6, 47 (1973)

[8] A. Gailitis, Mag. Gidrodyn. 32, 63 (1996)

[9] F. Stefani, G. Gerbeth, A. Gailitis, in Transfer Phenomena in Magnetohydrodynamic and Electroconducting Flows, edited by A. Alemany, Ph. Marty, P. Thibault, (Kluwer Academic Publishers, Dordrecht, 1999), p. 31

[10] M. Christen, H. Hänel, and G. Will, in Beiträge zu Fluidenergiemaschinen, Bd. 4, (Verlag und Bildarchiv W. H. Faragallah, Sulzbach/Ts. 1998), p. 111

[11] A. Gailitis, B. G. Karasev, I. R. Kirillov, O. A. Lielausis, A. P. Ogorodnikov, in Liquid Metal Magnetohydrodynamics, edited by J. Lielpeteris and R. Moreau, (Kluwer Academic Publishers, Dordrecht, 1989), p. 413 